\title{
Fragility index of meta-analyses in paediatric surgery
}

\author{
Priyam Saikia $^{1}\left[\right.$ B $\cdot$ Bandana Thakuria ${ }^{1}$
}

Accepted: 26 April 2021 / Published online: 10 May 2021

(c) The Author(s), under exclusive licence to Springer-Verlag GmbH Germany, part of Springer Nature 2021

To the editor,

We are doing a few projects on fragility index of randomized controlled trials (RCTs) and meta-analyses. Therefore, we read the work by Arne Schröder and colleagues with great enthusiasm [1]. Ignacio Atal and colleagues described the fragility index of meta-analyses that include only RCTs reporting dichotomous outcome [2]. Though the authors did not clearly state that the index proposed by them should/ cannot be used for meta-analyses that combines studies of different methodologies, their subsequent analysis did not include such meta-analyses [2]. We also believe that it should not be used for meta-analyses that includes studies other than parallel arm RCTs with 1:1 patient allocation. Unfortunately neither the search strategy used by Schröder A et al. nor the list of included meta-analyses has been mentioned in the published manuscript. Therefore, we could not refrain from assuming that the included meta-analyses may not have been appropriate to be used for the methodology used for calculating the fragility index. We are more worried about the validity of the analysis by Schröder et al. as they themselves mention that "randomised-controlled trials are scarce in paediatric surgery" and "evidence is often drawn from other study types and their meta-analyses in particular"!

Arne Schröder and colleagues have included meta-analyses that reports statistically significant result, whereas the online tool used by them can also be used for meta-analyses of RCTs reporting statistically non-significant results. It would have been interesting to know the fragility index of such meta-analyses too.

\section{References}

1. Schröder A, Muensterer OJ, Oetzmann von Sochaczewski C (2021) Meta-analyses in paediatric surgery are often fragile: implications and consequences. Pediatr Surg Int. https://doi.org/ 10.1007/s00383-020-04827-5

2. Atal I, Porcher R, Boutron I, Ravaud P (2019) The statistical significance of meta-analyses is frequently fragile: definition of a fragility index for meta-analyses. J Clin Epidemiol 111:32-40. https://doi.org/10.1016/j.jclinepi.2019.03.012

Publisher's Note Springer Nature remains neutral with regard to jurisdictional claims in published maps and institutional affiliations.
Priyam Saikia

saikia.priyam80@gmail.com

1 Gauhati Medical College and Hospital, Guwahati, Assam, India 